\title{
Giant Inguino-scrotal swelling
}

\author{
Meghna Kinjalk* \\ Department of General Surgery, Mahatma Gandhi Mission's Medical college, India
}

\section{Brief history}

A 51-year-old gentleman presented to outpatient department with complains of swelling in left inguinoscrotal region for 6 years. The swelling was insidious to begin with, and gradually progressed to present size. The swelling used to disappear on lying down and appeared on standing position, but since past 1 year, swelling did not disappear on lying down. He also complained of dragging pain in the groin for 2 months. There was no history of chronic constipation, chronic cough, vomiting or any urinary complains (Figure 1).

No history of any co morbidity. No history of appendectomy or any other surgeries in the past. No habits.

Patient was examined in standing as well as lying down position.

On Inspection, single, large pear-shaped swelling, approximately $18^{\star} 10 \mathrm{~cm}$ was seen, extending from the left inguinal region to the base of scrotum. Skin over the swelling was normal, no sinuses/scars seen.

On palpation, no local rise of temperature and no tenderness was present in the swelling. Deep Ring Occlusion test could not be done as swelling was irreducible. Could not get above the swelling.

\section{Swelling was dull on percussion.}

The contralateral side and external genitalia were normal. Per rectal examination was normal. There were no scars or lump in the abdomen.

\section{Case discussion}

The diagnosis at the end of history could have been from a hydrocoele/chylocoele to Inguinal hernia. After combining clinical examination and history, the diagnosis of Inguinal hernia was formed. The complications of Inguinal hernia like obstruction (vomiting, inability to pass flatus/stools), strangulation (tenderness, local rise of temperature along with obstructive symptoms) was ruled out.

Based on the history and clinical examination, a probable diagnosis of Left sided Irreducible complete inguinal hernia was made. Surgical management was planned. Intraoperative finding: An Indirect inguinal hernia with content being omentum. Lichtenstein Tension - free Hernioplasty was performed (Figure 2).

Copyright: (C2019 Kinjalk M. This is an open-access article distributed under the terms of the Creative Commons Attribution License, which permits unrestricted use, distribution, and reproduction in any medium, provided the original author and source are credited.

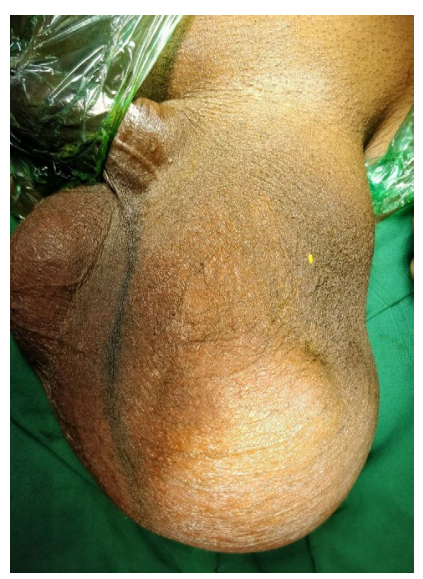

Figure 1. Left inguinoscrotal swelling

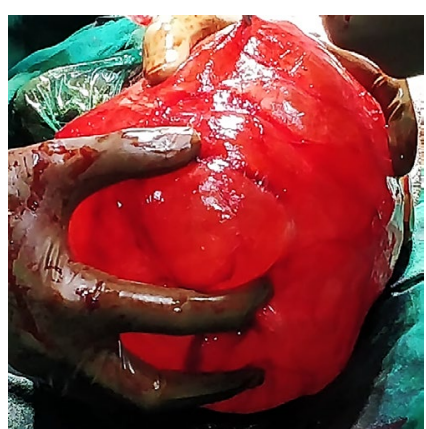

Figure 2. Intraoperative finding showing large hernial sac in a case of Indirect Inguinal hernia

\section{Conclusion}

Inguinal hernia, as the name suggests and commonly presents as a small inguinal swelling and usually patient presents at an initial stage. But as it is said that the "No two human will have the exact same anatomical arrangement throughout their body." Similarly, the case presentation for a diagnosis can vary, it's the history and clinical examination which helps to solve the mystery and give us a clinical diagnosis which can be later supported by Imaging modalities.

${ }^{*}$ Correspondence to: Meghna Kinjalk, Department of General Surgery, Mahatma Gandhi Mission's Medical college and hospital Sector 1 and 2, Near Sion-Panvel Highway, Kamothe-410209, Navi Mumbai, Maharashtra, India, Tel: 9167985006; E-mail: mkinjalk@gmail.com

Received: August 08, 2019; Accepted: August 21, 2019; Published: August 26, 2019 